\title{
LIPID PROFILE IN PSORIASIS
}

\author{
Poudyal $Y^{1}$, Rajbhandari SL ${ }^{2}$
}

\section{ABSTRACT}

INTRODUCTION: Psoriasis is a common inflammatory skin disorder. A predisposition to vascular occlusive disease and high prevalence of heart disease has been seen to be associated with psoriasis and it has been suggested that some of these patients have some disorders of lipid metabolism. Dyslipidemia has been suggested in the pathogenesis of this phenomenon.

METHODS: One hundred and six patients were selected for the study. Among them 53 (male=26, female=27) with psoriasis were taken as case and 53 with other dermatologic diseases, after matching for age and sex, were taken as control. The lipid profile that comprised four parameters: serum HDL, LDL-C, TC and TG were determined for both case and control and compared.

RESULTS: It was found that serum TG, TC levels were significantly higher and serum $\mathrm{HDL}$ level was significantly lower in patients with psoriasis $(\mathrm{P}<0.05)$. When the risk factors like alcoholism and smoking were adjusted, only abnormal serum level of TG and TC were seen to be significantly associated with psoriasis.

CONCLUSION: This study showed that abnormal TG and TC are associated with psoriasis. This fact may be responsible for the high prevalence of cardiovascular events in psoriasis. It may be useful to do early screening and treatment of dyslipidemia to prevent the adverse cardiovascular event.

\section{KEYWORDS: Psoriasis, Dyslipidemia, Lipid, Heart disease}

1. Assistant Professor, Department of Dermatology, Universal College of Medical Sciences \& Teaching Hospital, Bhairahawa, Nepal

2. Professor, Department of Dermatology, Universal College of Medical Sciences \& Teaching Hospital, Bhairahawa, Nepal

\author{
For correspondence \\ Dr. Yogesh Poudyal \\ Assistant Professor, \\ Department of Dermatology, \\ Universal College of Medical Sciences \& Teaching Hospital \\ Bhairahawa, Nepal \\ Email: neverland791@yahoo.com
}




\section{INTRODUCTION}

Psoriasis is a common inflammatory skin disorder clinically characterized by sharply demarcated chronic erythematous plaques, covered by silvery white scales, preferentially at the elbow, knee, scalp, umbilicus and lumbar area. ${ }^{1}$ Examination of available population based studies reveal prevalence ranging from $0.2 \%$ to $4.8 \%{ }^{2}$

Patients with psoriasis appear to have an increased morbidity and mortality from cardiovascular events, especially those with a severe and long duration of psoriasis. However, the pathogenesis of atherothrombotic events in psoriasis patients remains to be established. Multiple factors including aberrant lipid and lipoprotein profiles, increased oxidative stress, decreased antioxidant capacity and other established risk factors such as hypertension, obesity and diabetes mellitus have been associated with psoriasis. ${ }^{3}$

Alcoholism and smoking are the important risk factors for coronary heart disease. It has been shown that smoking and alcoholism are also important risk factor for psoriasis. ${ }^{4}$ Dyslipidemia is another important cardiovascular risk factor. Studies have suggested the hypothesis that psoriasis may be a predisposing factor to occlusive vascular episode. ${ }^{5}$

We conducted this study to show that like other risk factors common to heart disease and psoriasis, serum lipid abnormality may be associated with psoriasis risk.

\section{MATERIALSAND METHODS}

The study was designed as a case control study. The total number of patients were one hundred and six, among them 53 were case and 53 were control. The study subjects were taken from the dermatology OPD of Bir Hospital, Mahabauddha, Kathmandu and Shree Birendra Hospital, chhauni, Kathmandu. The age and sex were matched.

The approval of the study was obtained from the subject committee and Institutional review board, National Academy of Medical Sciences (NAMS), Bir Hospital. The period of this study was one year from January 2010 to December 2010.

Any patients presenting with Chronic Plaque type, Pustular and Guttate psoriasis were included as case. Patients with other skin disease were taken as control. The exclusion criteria were: those with the family history of hypertension and hyperlipidemia; patients on medications like beta blockers, oral corticosteroid, lipid lowering agents, thiazide, oral retinoids, patients with known history of diabetes, hypothyroidism, nephrotic syndrome, obstructive liver disease and connective tissue disease, patients below 16 years and above 60 years.

A written informed consent was taken after explaining all the relevant details and its importance. Those who did not give consent for any reasons were excluded from the study. Confidentiality was maintained.

The venous blood samples were taken from the patient after overnight fast. After collecting blood, it was allowed to clot and serum was used for detection of SerumTriglyceride (TG), Total cholesterol (TC), High density lipoprotein (HDL) and Low density Lipoprotein (LDL).The determination of TG, TC and HDL was done through the auto- analyser (ERBA diagnostic Mannheim $\mathrm{GmbH}$, Germany) through enzymatic colorimetric assay. LDL was calculated manually in lab from the formula: $\mathrm{LDL}-\mathrm{C}=\mathrm{TC}-(\mathrm{TG} / 5+\mathrm{HDL})$.

All the patients were diagnosed clinically and the severity of psoriasis was assessed by PASI score. ${ }^{6}$ Demographic and other data were recorded in performa through questionnaire and lab report. BMI was calculated by the formula:

$\mathrm{BMI}=$ weight in $\mathrm{Kg} / \mathrm{square}$ of height in meters.

Microsoft excel and SPSS statistics Ver.17 were used for recording and analyzing data. Descriptive statistics methods like central tendency calculation, deviation/dispersion calculation were worked out. Fisher's exact test, Chi-square test, independent $t$ test, correlation analysis were used. Level of significance ( 0.05 ) was predetermined and $\mathrm{CI}$ (confidence interval) of $95 \%$ was constructed.

\section{RESULTS}

The total number was 106 , among them $49.1 \%$ were male and $50.9 \%$ were female. Three types of psoriasis were included in this study (table1). The maximum number was Chronic Plaque type. Most of the patients have a chronic course of more than 2 years duration reflecting the fact that psoriasis is a chronic disease. Those diseases taken as control were melasma, acne, superficial skin infection, urticaria. 
Table 1: Gender wise distribution of different types of psoriasis

\begin{tabular}{|l|c|c|c|}
\hline \multirow{2}{*}{ Types of psoriasis } & \multicolumn{2}{|c|}{ Number of patients } & \multirow{2}{*}{ Total } \\
\cline { 2 - 3 } & Male & female & Total \\
\hline Chronic Plaque & 23 & 23 & 46 \\
\hline Pustular & 1 & 0 & 1 \\
\hline Guttate & 2 & 4 & 6 \\
\hline Total & 26 & 27 & 53 \\
\hline
\end{tabular}

Smoking and alcoholism are two important risk factors associated with psoriasis. Significant association was found between smoking and psoriasis (Table 2).

Table 2: The prevalence of risk factor in case and control

\begin{tabular}{|l|l|l|l|l|}
\hline Risk factor & Case & Control & Total & P-value \\
\hline Smoker & $17(32.1 \%)$ & $8(15.1 \%)$ & 25 & 0.033 \\
\hline Non-Smoker & $36(67.9 \%)$ & $45(84.9 \%)$ & 81 & \\
\hline Alcoholics & $12(22.6 \%)$ & $8(15.1 \%)$ & 20 & 0.299 \\
\hline Non- Alcoholics & $41(77.4 \%)$ & $45(84.9 \%)$ & 86 & \\
\hline Total & 106 & 106 & 106 & \\
\hline
\end{tabular}

In the clinical and demographic characteristics of patients like age, height, weight, BMI and BP, no significant difference were found. PASIranged from 2 to 29.38.( Table 3 )

Table 3: The clinical and demographic characteristics

\begin{tabular}{|l|l|l|l|}
\hline Characteristics & Case* & Control* $^{*}$ & P-value \\
\hline Age (years) & $34.67 \pm 12.60$ & $34.67 \pm 12.60$ & 1 \\
\hline Height (mtr) & $1.60 \pm 0.063$ & $1.61 \pm 0.075$ & 0.607 \\
\hline Weight (Kg) & $61.094 \pm 11.09$ & $60.528 \pm 9.512$ & 0.779 \\
\hline BMI (Kg/m2) & $23.84 \pm 3.81$ & $23.29 \pm 2.447$ & 0.382 \\
\hline BP(mmHg) & $113.24 / 69.15 \pm 12.24 / 6.99$ & $116.24 / 69.20 \pm 11.57 / 6.14$ & \\
\hline PASI & $10.47 \pm 6.95$ & & $0.121 / 0.965$ \\
\hline
\end{tabular}

${ }^{*}$ mean \pm standard deviation

The number of dyslipidemia noted in case and control is shown in table 4 . There was a statistically significant association of abnormality in TG, TC and $\mathrm{HDL}$.
Table 4: Dyslipidemia and psoriasis

\begin{tabular}{|l|c|c|c|c|c|}
\hline \multirow{2}{*}{ Lipid } & \multicolumn{2}{|c|}{ Case } & \multicolumn{2}{c|}{ Control } & \multirow{2}{*}{ P-value } \\
\cline { 2 - 5 } & Normal & Abnormal & Normal & Abnormal & \\
\hline TG & 32 & 21 & 46 & 7 & 0.002 \\
\hline TC & 30 & 23 & 40 & 13 & 0.032 \\
\hline HDL & 44 & 09 & 53 & 0 & 0.001 \\
\hline LDL & 49 & 04 & 53 & 0 & 0.059 \\
\hline
\end{tabular}

To find the relation between PASI score and associated abnormalities in lipid parameters, the data was analysed and recorded and no significant association between severity of psoriasis and lipid abnormality was noted. Similarly correlation analysis was done and it showed weak correlation between PASI score and HDL $(r=179)$.

Lipid abnormality was also seen in smoker and alcoholics. Considering smoking and alcoholism as confounding variables, without adjusting for these factors odd ratio for TG and TC were 4.086 and 2.566 indicating abnormal TG and TC and is approximately 4 and 2.5 times likely to be associated with psoriasis. Since there were no abnormal lipid values for HDL and LDL in control the odd ratio was not calculated.

\section{DISCUSSION}

The study showed that abnormal lipid profile is associated with psoriasis. Among four lipid parameters that were considered in study only three parameters TG, TC and HDL are associated without adjusting for the risk factors.

The result is consistent with the studies done in the past. The study of Piskin ${ }^{7}$ showed association of LDL and TC. Dratlen's study $^{8}$ showed only abnormal HDL is associated with psoriasis. The study by Akhyami ${ }^{9}$ showed abnormal TC, TG and LDL but not HDL to be associated with psoriasis. In the study done by Malbris ${ }^{3}$ only abnormal HDL was associated with psoriasis. The study by Aldhalimi ${ }^{10}$ showed association of only LDL,TC and TG with psoriasis. As in our study most studies showed association of abnormal TG with psoriasis. Abnormal LDL though found to be associated in other few studies, was not found in our study. This indicates that our study supports the evidence that abnormal serum lipids is associated with psoriasis.

After adjusting the risk factors like smoking and alcohol, only association of abnormal level of TC and TG was seen with psoriasis. Malbris et $\mathrm{al}^{3}$ showed that without adjusting for the confounding variables abnormal HDL and TC were associated 
with psoriasis, but after adjustment only HDL was associated. This shows that confounding variables like smoking and alcoholism need to be considered in psoriatic patients as these factors may lead to hyerlipidemia.

Our study showed no relation between disease severity and abnormal lipid profile, but weak correlation between HDL and severity of psoriasis. Study of Malbris ${ }^{3}$ showed no correlation but Gisondi et al ${ }^{11}$ showed significant correlation between severity of psoriasis and triglyceridemia. There are conflicting and limited study between severity of psoriasis and associated dyslipidemia. Further study is needed in this regard.

Elevated serum TG and lowserum HDL have been reported to be risk factors for the occlusive vascular disease. Patients with psoriasis have known microvascular abnormalities in both affected and unaffected skin. The reason for the changes in lipid metabolism in psoriasis patients have not been satisfactorily explained, since psoriasis is influenced by disorder in genetic alteration which may play a role for the dyslipidemia. $^{8}$

\section{CONCLUSION}

Abnormal serum lipid profile is seen to be associated with psoriasis, it is important to note that psoriasis may not only be disorder of skin; systemic involvement should also be considered.

\section{REFERENCES}

1) Schon M, Boehncke WH. Psoriasis-immunopathogenesis, genetics and therapeutic perspective. $N$ Eng $J$ Med 2005, 352:1899-1912.PMid:15872205

2) Gudjonsson JE, Elder JE. Psoriasis epidemiology. Cl Dermatol 2007;25:535-546. http://dx.doi.org/10.1016/j.clindermatol. 2007.08.007PMid:18021890

3) Malbris L, Granath F, Hamsten A, Stahle M. Psoriasis is associated with lipid abnormalities at the onset of skin disease. JAm Acad Dermatol 2006; 54:614-621.

http://dx.doi.org/10.1016/j.jaad.2005.11.1079PMid:16546581
4) Qureshi AA, Dominguez PL, Choi HK, Han J, Curhan G. Alcohol intake and risk of incident psoriasis in US woman. Arch Dermatol 2010; 146(12):1364-1369.

4) Qureshi AA, Dominguez PL, Choi HK, Han J, Curhan G. Alcohol intake and riskof incident psoriasis in US woman. Arch Dermatol 2010; 146(12):1364-1369.

http://dx.doi.org/10.1001/archdermatol.2010.204PMid:2071 3772 PMCid:PMC3017376

5) Mc Donald CJ, Calabresi P. Psoriasis and occlusive vascular disease. BrJDermatol 1978; 99:469-475. http://dx.doi.org/10.1111/j.1365-2133.1978.tb02012.x

6) Okum MM. Psoriasis Area Severity index: Nuts and bolts of measuring disease severity in psoriasis. Clin Dermatol 2008; 26:653-656.

http://dx.doi.org/10.1016/j.clindermatol.2008.08.002PMid:1 8940548

7) Piskin S, Gurkok F, Ekuklu G, Senol M. Serum lipid levelsin psoriasis. Yon MedJ2003; 44(1):24-26.PMid:12619171

8) Drateln CR, Martinez-Abundis E, Balcazar-Munoz BR, Bustos-Saldana R, Gonzalez-Ortiz M. Lipid profile, insulin secretion and insulin sensitivity in psoriasis. J Am Acad Dermatol 2003;48:882-885.

http://dx.doi.org/10.1067/mjd.2003.446PMid:12789179

9) Akhyani M, EhsaniAH, Robati RM,RobatiAM. The lipid profile in psoriasis: a controlled study. $J$ Eur Acad Dermatol Ven 2007;21:1320-1332.http://dx.doi.org/10.1111/j.14683083.2007.02260.xPMid:17958837

10) Aldhalimi MA, Almuhanna SJ, Alrikabi SH. Serum lipid level in Iraqi patients with psoriasis. Skin med 2010;8(4):204206.PMid:21137604

11) Gisondi P, Tessari G, Conti A, Piaserico S, Schianchi $S$, Peserico A et al. Prevalence of metabolic syndrome in patients with psoriasis: a hospital based case control study. $\mathrm{Br} J$ Dermatol 2007; 157:68-73.http://dx.doi.org/10.1111/j.13652133.2007.07986.xPMid:17553036 